\title{
Litiasis biliar en el lactante. A propósito de un caso
}

\author{
MN. Fernández Martínez ${ }^{\mathrm{a}}$, J. Ramallo Hermo ${ }^{\mathrm{a}}$, \\ MV. González Conde ${ }^{b}$, MS. Cameán Hermo ${ }^{c}$ \\ apediatra. CS de Boiro. A Coruña. España. \\ bPediatra. CS de A Cañiza. Pontevedra. España. \\ CEnfermera de Pediatría. CS de Boiro. A Coruña. España.
}

Fecha de publicación en Internet: 12 de noviembre de 2010

\section{Resumen}

Hasta hace poco, la litiasis biliar en el niño se consideraba una enfermedad infrecuente. El número de casos diagnosticados ha aumentado mucho en los últimos años y esto se debe, principalmente, al uso de la ecografía como técnica de estudio ante todo cuadro de dolor abdominal recurrente o inespecífico. La litiasis biliar sintomática en lactantes cursa como un cuadro de molestias abdominales inespecíficas, por lo que es obligado tenerlo presente en el diagnóstico diferencial de todo lactante con evolución desfavorable a pesar del tratamiento de otras patologías más frecuentes.

Palabras clave: Litiasis biliar. Lactante.

Gallstones in the infant. A case report

Abstract

Until recently gallstones in baby was considered a rare disease. The number of diagnosed cases has risen much in recent years, this is mainly due the ultrasound as technique to study all recurrent abdominal pain or unspecific. The symptomatic gallstones in infant presents a table of nonspecific abdominal discomfort and it is bound to have it in the differential diagnosis of any infant with unfavorable evolution despite treatment of other diseases more frequently.

Key words: Gallstones. Infant.

\section{Introducción}

Hasta hace pocos años, la litiasis biliar en el niño se consideraba una enfermedad infrecuente ${ }^{1}$. En la infancia, dicha patología presenta peculiaridades diferentes a las del adulto. Muchos pacien- tes cursan de forma asintomática (aproximadamente el $50 \%$ ), por lo que frecuentemente no son diagnosticados hasta pasar la edad pediátrica².

En los últimos años ha aumentado el número de casos diagnosticados y esto

María Nieves Fernández Martínez, nievesfer@mundo-r.com

Los autores declaran no presentar conflictos de intereses en relación con la preparación y publicación de este artículo. 
se debe a diferentes motivos: un mayor índice de sospecha por parte del pediatra; la identificación de factores de riesgo de litiasis; mayor supervivencia de niños con patologías que predisponen a la litiasis biliar; y por último, y el más importante, por el uso cada vez más frecuente de la ecografía como técnica de estudio ante todo cuadro de dolor abdominal recurrente $o$ inespecífico ${ }^{3}$.

\section{Caso clínico}

Lactante varón de 18 meses de edad. Como antecedentes familiares presenta un hermano de 5 años diagnosticado de colon irritable y reflujo gastroesofágico. Nace por parto espontáneo de vértice y a término. Peso al nacer 3450 g. Cribados neonatales ecográfico, auditivo y metabólico normales.

Recibe lactancia materna exclusiva hasta los 5 meses, presentando un desarrollo pondoestatural adecuado hasta esa edad (peso y talla en P25). Coincidiendo con la introducción de lactancia artificial inicia un cuadro de vómitos alimentarios y digestiones lentas. Ante la sospecha de reflujo gastroesofágico se inicia tratamiento con omeprazol y domperidona, siendo la evolución desfavorable, por lo que se cambia la leche a un hidrolizado de proteínas y se deriva a la consulta de Gastroenterología pediátrica a los 7 meses de vida. La pHmetría es normal y se descarta alergia a proteínas de leche de vaca. Persiste la mala evolución clínica.

La curva de percentiles en peso y talla desciende hasta situarse entre P10 y P3 y al mismo tiempo se convierte en un paciente hiperfrecuentador de los Servicios de Urgencias.

A los 12 meses ingresa por un cuadro de gastroenteritis aguda y desnutrición grado I. Entre las múltiples pruebas diagnósticas se realiza una ecografía abdominal que es informada de adenopatías mesentéricas hasta de $12 \mathrm{~mm}$. Se le realiza una nueva pHmetría, que resulta normal, y un tránsito digestivo superior, informado como "intenso reflujo durante la exploración".

Al alta recibe alimentación con mezcla hipercalórica, omeprazol y domperidona. Persiste la mala evolución y reingresa a los 18 meses por un cuadro de vómitos sin tolerancia oral en los días previos.

Llama la atención, en la exploración física, el tinte subictérico de la piel. El estudio bioquímico resulta normal, pero se vuelve a solicitar una nueva exploración ecográfica haciendo hincapié en el cuadro de vómitos junto con el tinte subictérico de la piel. La ecografía pone en evidencia, a nivel de la vesícula biliar, 
2 cuerpos ecogénicos que producen sombra acústica compatible con litiasis biliar. Tras la colecistectomía laparoscópica, el niño inicia un cuadro de recuperación pondoestatural junto con la desaparición de los vómitos y el resto del cortejo sintomático acompañante.

\section{Discusión}

La litiasis biliar podría definirse como la presencia de cálculos en la luz de las vías biliares.

Predominan en la infancia los cálculos pigmentarios (80\%), correspondiendo a cálculos mixtos un 19\% aproximadamente y siendo excepcional la presencia de cálculos de colesterol puros $(<1 \%)^{2}$.

Hasta hace pocos años la litiasis biliar en la infancia se consideraba una enfermedad infrecuente y se asociaba fundamentalmente a enfermedad hemolítica.

Una evidente mejora en los métodos diagnósticos condiciona actualmente un aumento de la prevalencia relacionada, además, con una mayor supervivencia de patologías graves diagnosticadas y tratadas en los primeros meses de vida. Grandes prematuros, enterocolitis necrosante, sepsis y situaciones que requieren ayuno prolongado y nutrición parenteral, han demostrado que la prevalencia no es tan escasa.
El estudio de Palsciano et al. ${ }^{4}$, realizado en niños italianos de 6 a 9 años, aparentemente sanos y asintomáticos, encontró una incidencia de litiasis biliar del 0,13\%. La incidencia aumentaba con la edad y en los adultos era más frecuente en mujeres.

Podemos afirmar que, en un $50 \%$ de los casos, no se encuentra en la infancia factor etiológico que justifique la litiasis.

Friesen $^{5}$, en una serie de 698 niños, objetivaba que en menores de un año los cálculos eran idiopáticos en un $37 \%$ de los casos, mientras que solo lo eran en un $4 \%$ en pacientes entre 6 y 11 años.

Determinadas enfermedades predisponen al desarrollo de litiasis biliar: enfermedades hemolíticas, enfermedades hepáticas y enfermedades intestinales.

Ruibal et al. ${ }^{6}$ analizaban un total de 123 niños procedentes de distintos hospitales. El $64 \%$ de los cálculos eran idiopáticos, el 15\% por enfermedad hemolítica, el $7 \%$ por obesidad y el $12 \%$ por otras enfermedades.

Friesen y Roberts ${ }^{7}$, en un estudio etiológico de 693 niños, en correlacción con la edad encontraron que en lactantes por debajo de los 12 meses, el 37\% de los casos eran idiopáticos.

Podemos afirmar entonces, que en un elevado porcentaje de pacientes se llega al diagnóstico como hallazgo casual. 
Las manifestaciones clínicas se pueden dividir en 3 apartados: síntomas biliares (cólico biliar e ictericia), síntomas inespecíficos (dolor abdominal inespecífico, flatulencia o plenitud post-prandial) y el grupo de la litiasis asintomática.

Las manifestaciones clínicas de la litiasis en niños suelen ser muy poco específicas, sobre todo en lactantes.

Habíamos reseñado anteriormente que la incidencia de la litiasis biliar en la infancia había aumentado, sobre todo debido a que el dolor abdominal recurrente en niños suele ser estudiado ecográficamente $^{8}$.

Y aún podemos llegar a más afirmando que todo cuadro de dolor abdominal inespecífico en niños, y sobre todo en lactantes, con evolución tórpida a pesar del tratamiento de un posible diagnóstico frecuente (como pueden ser el reflu- jo gastroesofágico o una alergia a proteínas de leche de vaca), ha de ser estudiado ecográficamente, pensando en la litiasis como una posible causa del cuadro clínico.

En cuanto al tratamiento, podemos decir que la litiasis asintomática, de hallazgo casual, sin enfermedad de base ni factores de riesgo asociados, no precisa en principio tratamiento. Se realizarán revisiones periódicas valorando un cambio de actitud si se hace sintomática ${ }^{9}$.

El tratamiento definitivo de la litiasis sintomática, salvo en determinadas situaciones, consiste en la colecistectomía laparoscópica ${ }^{10}$.

Conclusiones: es obligado descartar la existencia de litiasis biliar en cualquier niño con síntomas abdominales inespecíficos y de mala evolución a través de un estudio ecográfico exhaustivo.

\section{Bibliografía}

1. Escobar H, García MD, Olivares P. Litiasis biliar en la infancia: actitudes terapéuticas. An Pediatr (Barc). 2004;60:170-4.

2. Díaz C. Colelitiasis. Pediatr Integral. 2007;XI(3):257-63.

3. Carbajo AJ. Litiasis biliar. Hepatología. En: Protocolos diagnósticos y terapéuticos de gas- troenterología, hepatología y nutrición en Pediatría. Barcelona: Asociación Española de Pediatría; 2002. p. 263-7.

4. Palasciano $G$, Portincasa $P$, Vinciguerra $V$, Velardi A, Tardi S, Baldassarre G, et al. Gallstone prevalence and galbladder volume in children and adolescents; an epidemiological ultrasonographic survey and relationship to mass index. Am J Gastroenterol. 1989;84:1378-82. 
5. Friesen C, Roberts C. Cholelithiasis; Clinical characteristics in children. Clin Pediatr. 1989;28: 194-8.

6. Ruibal JL, Aleo E, Álvarez A, Piñeiro E, Gómez-Casares R. Colelitiasis en la infancia: análisis de 24 pacientes y revisión de 123 casos publicados en España. An Esp Pediatr. 2000;54: 120-5.

7. Friesen CA, Roberts CC. Cholelithiasis. Clinical characteristics in children. Case analysis and literature review. Clin Pediatr. 1989;28: 294-8.

8. Wacholder V, Hiams L, Questa H, Vailone $P$, Bailez M, Sasson L. Litiasis biliar en la infancia. Rev Cir Infantil. 1991;1:17-8.

9. Lobe TE. Cholelithiasis and cholecystitis in children. Semin Pediatr Surg. 2000;9:170-6.

10. Mitenburg DM, Shaffer R, Breslin T, Brandt $M L$. Chaging indications for pediatric cholecystectomy. Pediatrics. 2000;105:1250-3. 